\title{
Correlations And Correlates Of Post-Traumatic Growth And Post-Traumatic Stress Symptoms In Patients With Breast Cancer
}

This article was published in the following Dove Press journal:

Neuropsychiatric Disease and Treatment

\author{
Hong-Ming Chen ${ }^{1,2, *}$ \\ Vincent Chin-Hung Chen ${ }^{1,2, *}$ \\ Han-Pin Hsiao' \\ Yi-Ping Weng' \\ Ya-Ting Hsu' \\ Jun-Cheng Weng $\mathbb{D}^{1,3}$ \\ Jian-An Su ${ }^{1,2,4}$ \\ Yi-Lung Chen $\mathbb{D}^{5,6}$
}

'Department of Psychiatry, Chang Gung Medical Foundation, Chiayi Chang Gung

Memorial Hospital, Chiayi, Taiwan;

${ }^{2}$ School of Medicine, Chang Gung

University, Taoyuan, Taiwan;

${ }^{3}$ Department of Medical Imaging and Radiological Sciences, Chang Gung

University, Taoyuan, Taiwan;

${ }^{4}$ Department of Nursing, Chang Gung

Institute of Technology, Taoyuan, Taiwan;

${ }^{5}$ Department of Healthcare

Administration, Asia University, Taichung,

Taiwan; ${ }^{6}$ Department of Psychology, Asia

University, Taichung, Taiwan

*These authors contributed equally to this work

Correspondence: Yi-Lung Chen Department of Healthcare

Administration, Asia University, 500,

Lioufeng Road, Wufeng, Taichung 4I3,

Taiwan

Tel +886 423323456 Ext 20106

Fax +886 4233 I 6699

Email elong@asia.edu.tw

Jian-An Su

Department of Psychiatry, Chang Gung Medical Foundation, Chiayi Chang Gung Memorial Hospital, No. 6 West Sec.,

Chia-Pu Road, Puzi City, Chiayi 613,

Taiwan

Tel +8865362 1000 Ext 2855

Fax +886 53623002

Email jian.77I5@gmail.com
Purpose: Although post-traumatic growth (PTG) and post-traumatic stress symptoms (PTSS) might develop and coexist after a major trauma, few studies have simultaneously examined them in patients with breast cancer. This study investigated the correlation between PTG and PTSS and their differential correlates in patients with breast cancer.

Patients and methods: Overall, 145 patients with breast cancer were recruited. PTG and PTSS were assessed using the PTG inventory and the Chinese version of startle, physiological arousal, anger, and numbness, respectively. We investigated the effects of demographics, chemotherapy, depression, family support, alexithymia, and anxiety symptoms on PTG and PTSS. Multivariate linear regression analyses were performed to select the independent correlates of PTSS and PTG.

Result: An association was observed between PTG and PTSS $(r=0.21)$. Based on multiple regression models, the common correlate of PTG $(\beta=0.271)$ and PTSS $(\beta=0.212)$ was anxiety symptoms. Differential independent correlates were years of education $(\beta=0.272)$, receiving chemotherapy $(\beta=0.248)$, and family support $(\beta=0.259)$ for PTG, and chronic pain $(\beta=0.316)$ and poor cognition $(\beta=-0.350)$ for PTSS.

Conclusion: Differential correlates were observed for PTG and PTSS in patients with breast cancer. Possible mechanisms and relationships between PTG and PTSS were discussed.

Keywords: post-traumatic stress symptoms, post-traumatic growth, breast cancer, chemotherapy, family support

\section{Introduction}

Malignancy is a substantial societal burden worldwide and can substantially affect the mental health of patients because it is considered a traumatic event that implies a threat of death or severe physical injury and evokes psychological reactions, such as fear, avoidance, and hyperarousal. ${ }^{1}$ Notably, the diagnosis of life-threatening breast cancer is a significantly traumatic event. An increasing number of studies have reported that patients with cancer experience trauma-associated symptoms ${ }^{2,3}$ and that post-traumatic stress disorder (PTSD) was more often observed in cancer survivors than in the general population. ${ }^{4}$

A traumatic experience can conversely have a positive effect on patientsknown as post-traumatic growth (PTG). The concept of PTG was initially introduced by Tedeschi and Calhoun. ${ }^{5}$ They described the positive growth following a traumatic experience or disease, such as cancer. Growth in patients with cancer commonly involved the following aspects: new possibilities, relating to others, 
personal strength, spiritual change, and appreciation of life. ${ }^{5}$ PTG is the positive change that occurs as a result of the struggle with highly challenging life crises. ${ }^{6}$

The current relationship between PTG with PTSD remains inconsistent. Although some studies have reported regarding positive correlations between PTG with PTSD, ${ }^{7-10}$ other studies were unable to identify any association, ${ }^{11-15}$ and furthermore, inconsistent findings were observed in patients with cancer. ${ }^{11,12,16,17}$ Such inconsistent findings were probably due to differences in the type and stage of the diseases. Notably, PTSD was observed during the initial stages of breast cancer diagnosis, whereas PTG occurred when patients underwent treatment. ${ }^{17}$ Several factors have been reported to influence PTG and PTSD following trauma. ${ }^{18}$ Therefore, further research is necessary to investigate the manifestations of PTSD and PTG among different cancer populations. $^{16}$

Differentiating the correlates of PTSD and PTG is crucial because they correspond to the different responses to the traumatic experiences and ramifications of mental health. Furthermore, it is of merit to investigate PTSD and PTG in different cultures and ethnic groups because a previous study demonstrated that ethnic differences might influence PTSD and PTG. For example, AfricanAmerican patients with breast cancer were more likely to develop PTG than Caucasian women. ${ }^{19}$ Numerous studies have examined the potential risk factors for PTSD or associated symptoms after cancer diagnosis. ${ }^{11,12,16}$ Impaired psychosocial functioning, negative life events, health problems, and high emotional reactivity were considered predictors of PTSD. ${ }^{20}$ Anxiety symptoms, depression, health complaints, and sleep problems predicted intrusive thoughts after diagnosis. ${ }^{21,22}$ High prevalence of depression was observed in patients with breast cancer. ${ }^{23}$ Patients with stronger physical symptoms or side effects during treatment would have an increased risk of cancerrelated distress 4 months after treatment completion. ${ }^{24}$ In addition, PTG in patients with cancer was reported to be more likely in patients who had a higher education level; who were younger; whose diagnosis was pronounced a long time ago; who had a higher baseline level of illness intrusiveness; and who increased their social support, spirituality, and use of active adaptive coping strategies. ${ }^{25}$ Notably, chemotherapy may contribute to the traumatic experience in patients with cancer; however, its effect on PTSD and PTG is unclear. Nonetheless, the management of distress caused by cancer and its treatment remains a clinical challenge that warrants further exploration.
In this study, we highlighted the psychological effects seen in patients with breast cancer and simultaneously evaluated the positive and negative reactions, PTG, and post-traumatic stress symptoms (PTSS). In addition, we sought to determine the correlates to differentiate PTG and PTSS.

\section{Methods}

\section{Participants}

A convenient cross-sectional study was conducted at the oncology clinic of Chiayi Chang Gung Memorial Hospital from November 2017 to February 2019. We approached 359 potentially eligible participants aged $\geq 20$ years who were diagnosed with invasive breast cancer from stage 0 to 3 without evidence of metastasis. Exclusion criteria for the study participants were as follows: 1) any neurological disorder or a lifetime history of severe head trauma; 2) history of mental retardation, bipolar disorder, schizophrenia, and substance-related disorders within the preceding 12 months or past suicide attempt history; 3) illiteracy; 4) history of developmental delay; 5) severe visual impairment (e.g., cataract and glaucoma); and 6) current pregnancy. All participants were required to provide written informed consent. Eventually, with approximately 59\% refusal rate, 145 participants were included in this study. The primary reasons for refusal were unavailability or unwillingness to sign informed consent forms.

\section{Measures}

The demographic data of the participants were collected, including age, body mass index (BMI), years of education, marital status, and employment. Other categorical information was also collected, such as systemic treatments received (i.e., chemotherapy, radiation therapy, and hormone therapy); existing comorbidities (i.e., diabetes and hypertension); chronic pain and pain scores; psychiatric history; family history of psychiatric disease or breast cancer; and further detailed information to include cancer staging, procedure type if operated, cancer subtypes [i.e., lymph node involvement, estrogen receptor-positive breast cancer, progesterone receptor-positive breast cancer, and human epidermal growth factor receptor (HER2)-positive breast cancer], and gynecological health. Whether the participants received cancer treatment or not was per physicians' clinical judgment based on medical guidelines, including cancer stage and the result of Her2 examination. Regarding chemotherapy, the participants were more 
likely to receive neoadjuvant or adjuvant chemotherapy if they had early-stage cancer, whereas they were more likely to receive palliative chemotherapy for advanced-stage cancer. The following series of self-reported questionnaires were administered to all participants:

\section{Post-Traumatic Stress Symptoms}

The SPAN (startle, physiological arousal, anger, and numbness) questionnaire was a shorter version of the 17item version of the Davidson Trauma Scale. We used the Chinese version of SPAN (SPAN-C), which was validated in 2003. SPAN comprises 4 items to assess the frequency and severity of PTSS, using a four-point scale ranging from 0 (not at all distressing) to 4 (extremely distressing). A higher score indicated more severe PTSS. SPAN-C was reported to have satisfactory psychometric reliability and validity. ${ }^{26}$ This study observed an excellent internal consistency with a Cronbach's $\alpha$ of 0.90 for SPAN-C.

\section{Post-Traumatic Growth}

Post-traumatic Growth Inventory (PTGI) was developed to assess the growth experience after a traumatic event. PTGI has 21 items with the following 5 subscales: new possibilities, relating to others, personal strength, spiritual change, and appreciation of life and uses a 6-point scale response format ranging from 0 to 5 , with 0 indicating "I did not experience this change as a result of my crisis" and 5 indicating "I experienced this change to a very great degree as a result of my crisis". ${ }^{5}$ A higher score indicates a higher growth experience after trauma. This study observed an excellent internal consistency with a Cronbach's $\alpha$ of 0.94 for PTGI.

\section{Family Support}

Family support was examined using the family adaptation, partnership, growth, affection, and resolve (family APGAR), a 5-point self-report questionnaire tapping into the 5 dimensions of perceived family support. Each item was rated on a 3-point Likert scale: 0 for hardly ever, 1 for some of the time, and 2 for almost always. A higher score indicates better family function. An excellent internal consistency with a Cronbach's $\alpha$ of 0.86 was observed for family APGAR in this study.

\section{Alexithymia}

Alexithymia was examined using the Toronto Alexithymia Scale (TAS), a 20-item self-report questionnaire. The TAS is based on a 5 -point Likert scale, wherein $1=$ strongly disagree and $5=$ strongly agree. A higher score indicates more severe alexithymia. Satisfactory psychometric reliability and validity were reported. ${ }^{27}$ An excellent internal consistency with a Cronbach's $\alpha$ of 0.75 was observed for TAS in this study.

\section{Depression Symptoms}

The Patient Health Questionnaire-9 (PHQ-9) was designed to screen depression symptoms over the preceding 2 weeks and used a 4-point scale ranging from 0 (not at all) to 3 (nearly every day). The range of the PHQ is from 0 to 27, and a higher score of the PHQ indicates more severe depression symptoms. The PHQ is reported to have high reliability. An excellent internal consistency with a Cronbach's $\alpha$ of 0.75 was observed for PHQ in this study.

\section{Anxiety Symptoms}

The Hospital Anxiety and Depression Scale (HADS) was developed to examine anxiety symptoms and depression in people with physical health problems. The HADS comprises 14 items, 7 each for anxiety symptoms and depression and uses a 4-point scale. A higher score of the HADS indicates more severe depression and anxiety. The HADS has been suggested to be a reliable tool with satisfactory psychometric reliability and validity. In this study, we used only the anxiety symptoms section (HADS-A) to examine anxiety symptoms. This study observed an excellent internal consistency with a Cronbach's $\alpha$ of 0.85 for HADS-A.

\section{Fatigue}

The Brief Fatigue Inventory (BFI) is used to assess the effects of cancer-related fatigue. The BFI has 9 items with 2 different subscales (i.e., fatigue severity and fatigue interference) using an 11-point rating scale. The first 3 questions measure fatigue severity from 0 to 10 , with 0 indicating "no fatigue" to 10 indicating "as bad as you can imagine," and the other 6 questions assess fatigue interference with response options ranging from 0 to 10 , with 0 indicating "does not interfere," to 10 indicating "completely interferes." ${ }^{28}$ A higher score of the BFI indicates more severe cancer-related fatigue. An excellent internal consistency with a Cronbach's $\alpha$ of 0.86 was observed for the BFI in this study.

\section{Self-Perceived Health}

The EuroQol visual analog scale (EQ-VAS) is a standardized instrument for measuring generic health status, and we used it to assess the respondent's self-rated health (ranging from 0 to 100). A higher score indicates 
satisfactory self-perceived health. The validity and reliability of the EuroQol have been assessed for cancer. ${ }^{29}$

\section{Cognition}

The functional assessment of cancer therapy-cognitive (FACT-Cog) function is a self-report questionnaire designed to examine the cognitive function of patients with cancer for the 7 days before, during, and after chemotherapy. The FACT-Cog comprises 37 items and is based on a 5-point scale from 0 (never) to 4 (several times a day). A higher score indicates poorer cognition. The internal consistency with a Cronbach's $\alpha$ for the total score of FACT-Cog in this study was 0.82 . The FACT-Cog is considered to have satisfactory reliability, validity, and equivalence for clinical and research use. ${ }^{30}$

\section{Chronic Pain}

A VAS of chronic pain was used to determine participants' chronic pain on an 11-point scale ranging from 0 to 10 . A higher score indicates a higher self-perceived pain intensity. A previous study has demonstrated that the VAS of chronic pain is a simple, frequently used, reliable assessment tool for variations in pain intensity. ${ }^{31}$

Based on participants' energy and willingness, we further provided a short-form series of questionnaires, wherein SPAN-C, Family APGAR, PHQ-9, self-perceived health, FACT-Cog Function, chronic pain were included, but not PTGI, BFI, TAS, and HADS-A.

\section{Procedure}

This study was conducted in accordance with the Declaration of Helsinki and approved by the Institutional Review Board of Chiayi Chang Gung Memorial Hospital (approval number: 201700252B0C603). A psychiatric nurse and a research assistant, who had both earned a bachelor's degree in nursing and had extensive experience working in the field of psychiatry, were responsible for recruiting the participants, including identification of potential participant, initial contact, screening, and recruitment. Because of the convenience sampling in this study, the recruiters would identify and contact any potential eligible participant from inpatient and outpatient clinics. Informed consent was obtained from the participants after explaining the purpose and procedures of the study besides providing reassurance of confidentiality. Questionnaires were provided after obtaining the informed consent, and recruiters helped the participants to answer questionnaires if they had trouble understanding the concepts and contents of questionnaires.

\section{Statistical Analysis}

Statistical analyses were conducted using SAS 9.4 (SAS Institute Inc., Cary, NC, USA). Descriptive results were presented as the frequency and percentage for categorical variables and as the mean and standard deviation for continuous variables. Skewness and kurtosis were examined to examine the normality of continuous variables (i.e., all questionnaires). A correlation analysis with Pearson's $r$ was used to examine the association between PTG and PTSS. Two linear regression models were used to determine the correlates of PTG and PTSS with and without adjusting for the other. The unadjusted model was analyzed to present the crude associations of PTG and PTSS. The adjusted model was a multiple regression model with stepwise model selection, wherein all statistically significant correlates from the unadjusted model were added. The adjusted $R^{2}$ was used to quantify the explained variability of PTG and PTSS from the multivariate models. Variance inflation factor (VIF) was used to examine the severity of multicollinearity. O'Brien (2007) recommended that if VIF is greater than 5 , the regression coefficient may be inflated because of linear dependence with other predictors. ${ }^{32}$ Finally, the Durbin-Watson test was performed to examine whether the residuals from these regression models were independent or autocorrelated. An alpha level of 0.05 was considered significant for all analyses.

\section{Results}

Among 145 participants with breast cancer recruited in this study, 108 completed the full version of questionnaires, and 37 completed the short-form version. As shown in Table 1, the mean age and standard deviation for the participants were 52.25 and 10.45 , respectively. The mean BMI was $24.22(\mathrm{SD}=4.11)$, and the education level was 10.77 years $(\mathrm{SD}=3.95)$. Overall, $79.3 \%$ of the participants were married, with $51.8 \%$ being employed. Most of our participants were diagnosed as having stage $2(30.3 \%)$ or stage 3 (39.3\%) cancer and had experienced menopause (73.1\%). Most patients had undergone surgery (partial mastectomy: 78.6\%; total mastectomy: $20.7 \%$ ). The percentages of disease comorbidity were $18.6 \%$ for psychiatry disorders, $6.9 \%$ for diabetes, and $20.0 \%$ for hypertension. The percentage for systemic treatments were $26.2 \%$ for chemotherapy, $31.7 \%$ for radiotherapy, and $42.1 \%$ for hormone therapy. 
Table I Demographic Data, Health Condition, And SelfReported Inventory Of Patients With Breast Cancer $(\mathrm{N}=145)$

\begin{tabular}{|c|c|}
\hline Characteristics & Mean \pm SD/N (\%) \\
\hline Age, years & $52.25 \pm 10.45$ \\
\hline $\mathrm{BMI}, \mathrm{kg} / \mathrm{m}^{2}$ & $24.22 \pm 4.11$ \\
\hline Year of education & $10.77 \pm 3.95$ \\
\hline Marital status, married & II 5 (79.3) \\
\hline Employment & $75(51.7)$ \\
\hline Menopause Status (Menopause) & $106(73.1)$ \\
\hline \multicolumn{2}{|l|}{ Comorbidity } \\
\hline Psychiatric history & $27(18.6)$ \\
\hline Diabetes & $10(6.9)$ \\
\hline Hypertension & $29(20.0)$ \\
\hline \multicolumn{2}{|l|}{ Cancer stage $^{\star}$} \\
\hline 0 & $25(17.4)$ \\
\hline I & $44(30.6)$ \\
\hline 2 & $57(39.6)$ \\
\hline 3 & $18(12.4)$ \\
\hline \multicolumn{2}{|l|}{ Systemic treatments } \\
\hline Chemotherapy & $38(26.2)$ \\
\hline Radiotherapy & $46(3 I .7)$ \\
\hline Hormone therapy & $61(42.1)$ \\
\hline \multicolumn{2}{|l|}{ Family History } \\
\hline Breast Cancer & $30(20.7)$ \\
\hline Psychiatric disorders & $13(9.0)$ \\
\hline \multicolumn{2}{|l|}{ Surgical procedure $^{\star}$} \\
\hline Partial mastectomy & II4 (79.2) \\
\hline Total mastectomy & $30(20.8)$ \\
\hline \multicolumn{2}{|l|}{ Cancer subtypes } \\
\hline ER, positive & II4 (78.6) \\
\hline PR, positive & $97(66.9)$ \\
\hline Her2_neu, negative & $90(62.1)$ \\
\hline Her2_neu, positive & $25(17.2)$ \\
\hline Her2_neu, equivocal & $28(19.3)$ \\
\hline Lymph node involvement & $43(29.7)$ \\
\hline \multicolumn{2}{|l|}{ Self-report inventory } \\
\hline Family support (family APGAR score) & $6.26 \pm 3.16$ \\
\hline Depression (PHQ-9) & $4.79 \pm 4.47$ \\
\hline EQ-VAS & $71.91 \pm 19.00$ \\
\hline FACT-Cog, total score & $115.94 \pm 14.26$ \\
\hline PTSD (SPAN-C) & $0.77 \pm 2.01$ \\
\hline PTGI $(n=108)$, total scores & $32.54 \pm 26.17$ \\
\hline Chronic pain (score) & $1.37 \pm 2.24$ \\
\hline \multicolumn{2}{|l|}{ Fatigue $(\mathrm{BFI}, \mathrm{n}=108)$} \\
\hline Fatigue severity & $4.7 I \pm 6.42$ \\
\hline Fatigue interference & $3.31 \pm 5.63$ \\
\hline
\end{tabular}

(Continued)
Table I (Continued).

\begin{tabular}{|l|l|}
\hline Characteristics & Mean \pm SD/N (\%) \\
\hline Alexithymia (TAS, $n=108)$ & $55.31 \pm 5.90$ \\
Anxiety symptoms (HADS) $(\mathrm{n}=108)$ & $3.85 \pm 3.78$ \\
\hline
\end{tabular}

Note: ${ }^{\star}$ There was one missing piece of data on cancer stage and surgical procedure.

Abbreviations: $\mathrm{BFI}$, Brief Fatigue Inventory; ER, estrogen receptor; HADS-A, Hospital Anxiety and Depression Scale-Anxiety symptoms; Her2, human epidermal growth factor receptor; PHQ-9, Patient Health Questionnaire-9; PR, progesterone receptor; PTGI, Post-traumatic Growth Inventory; TAS, Toronto Alexithymia Scale; VAS, visual analog scale.

The percentages of participants who had a family history of breast cancer and psychiatric disorders were $9.0 \%$ and 20.7\%, respectively. The total mean PTGI score for PTG (21 items) was $32.54(\mathrm{SD}=26.17)$. The individual mean SPAN-C score for PTSS (4 items) was $0.77(\mathrm{SD}=2.01)$. Our variables were generally normally distributed with most items demonstrating no problematic levels of skewness (maximum skewness $=-0.98$ ) or kurtosis (maximum kurtosis $=1.391$ ), except for SPAN-C (skewness $=3.86$ and kurtosis $=17.20)$ and PHQ (skewness $=1.18$ and kurtosis $=$ 1.10). Because a positively skewed distribution was observed in SPAN-C (excess zero-count observation), we used the negative reciprocal transformation to reduce skewness. After log-transformation, the skewness and kurtosis were 0.95 and -0.87 , respectively, for SPAN-C and -0.27 and -0.67 , respectively, for PHQ - generally close to normal distribution. We observed a significant positive association between PTG and PTSS with Pearson's $r$ of 0.21 and a $p$-value of 0.032 (Figure 1).

For PTG, the results of the univariate analysis revealed that statistically significant crude associations were found between age, BMI, education, employment, diabetes, chemotherapy, family support, anxiety symptoms, and PTGI.

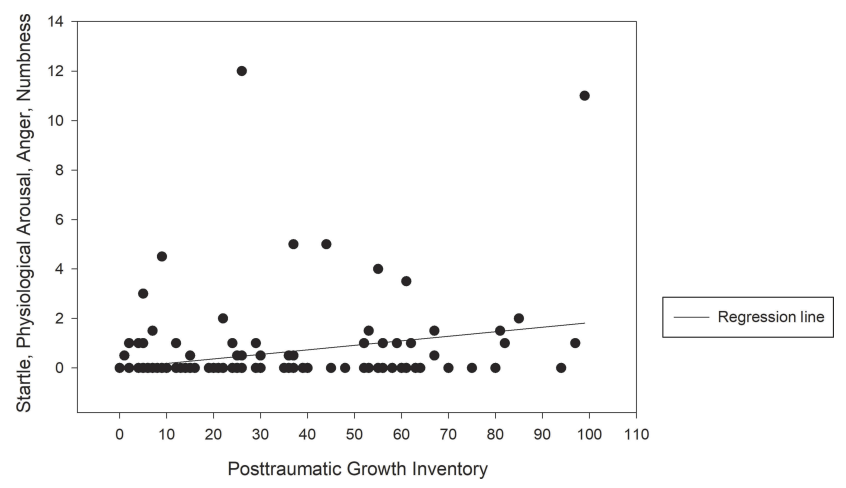

Figure I Scatter plots with a regression line of the correlations between posttraumatic stress symptoms and post-traumatic growth. 
Variables with statistical significance in the univariate analysis were entered into a multiple linear regression model, after being controlled for the other, and we found that only the years of education $(\mathrm{B}=1.852, \beta=0.272, p=0.003$ ), chemotherapy $(\mathrm{B}=13.615, \beta=0.248$, and $p=0.005)$, family support $(\mathrm{B}=2.106, \beta=0.259$, and $p=0.024)$, and anxiety symptoms $(\mathrm{B}=1.873, \beta=0.271$, and $p=0.035)$ preserved their independent associations in the PTGI (Table 2). This multivariate model had an adjusted $\mathrm{R}^{2}$ of 0.33 , suggesting that $33 \%$ of the variability of PTG was explained by years of education, chemotherapy, family support, and anxiety symptoms. The values of VIF in these models ranged from 1.02 to 1.24 , all of which were smaller than 2.5 recommended by O'Brien, thereby suggesting no obvious multicollinearity between variables in this model.
In addition, we did not observe a significant autocorrelation based on the Durbin-Watson test ( $p \mathrm{~s}>0.05)$.

Similar analyses were conducted for PTSS. We found that BMI, years of education, chronic pain, fatigue severity (BFI), alexithymia (TAS), depression symptoms (PHQ-9), anxiety symptoms (HADS-A), cognition (FACT-Cog total score), and psychiatric history were significantly associated with PTSS. Among risk factors identified using the unadjusted model, only chronic pain $(\mathrm{B}=0.049, \beta=0.316, p<$ $0.001)$, anxiety symptoms $(\mathrm{B}=0.018, \beta=0.212, p=0.011)$, and cognition $(\mathrm{B}=-0.009, \beta=-0.350, p<0.001)$ remained statistically significant after controlling for the others (Table 3). This multivariate model had an adjusted $\mathrm{R}^{2}$ of 0.40 , thereby suggesting that $40 \%$ of the variability of PTSS was explained by BMI, chronic pain, and anxiety

Table 2 Summary Of The Linear Regression Of Significant Predictors For PTGI In Patients With Breast Cancer

\begin{tabular}{|c|c|c|c|c|c|c|c|c|c|c|c|c|c|}
\hline \multirow[t]{2}{*}{ Variable } & \multicolumn{6}{|c|}{ Unadjusted Model } & \multicolumn{7}{|c|}{ Adjusted Model } \\
\hline & B & SE & $\boldsymbol{\beta}$ & p-value & $\mathbf{R}^{2}$ & DW & B & SE & $\boldsymbol{\beta}$ & p-value & VIF & $\mathbf{R}^{2}$ & DW \\
\hline Age, years & -0.057 & 0.029 & -0.210 & $0.031 *$ & 0.03 & 1.83 & - & - & & - & - & 0.33 & 1.87 \\
\hline BMI, $\mathrm{kg} / \mathrm{m}^{2}$ & -0.170 & 0.075 & -0.190 & $0.046 *$ & 0.03 & 1.78 & -1.080 & 0.538 & -0.165 & $0.075^{*}$ & 1.02 & & \\
\hline Year of education & 0.817 & 0.121 & 0.377 & $<0.001 * * *$ & 0.13 & 1.69 & 1.852 & 0.603 & 0.272 & $0.003^{* *}$ & 1.19 & & \\
\hline Diabetes & 0.167 & 0.052 & -0.221 & $0.0218^{*}$ & 0.04 & 1.80 & - & - & & - & - & & \\
\hline Chemotherapy & 0.174 & 0.057 & 0.256 & $0.0074 * *$ & 0.06 & 1.93 & 13.615 & 4.793 & 0.248 & $0.005^{* *}$ & 1.15 & & \\
\hline Hormone therapy & 0.333 & 0.064 & 0.223 & $0.0201 *$ & 0.04 & 1.81 & - & - & & - & - & & \\
\hline $\begin{array}{l}\text { Family support } \\
\text { (Family APGAR) }\end{array}$ & 0.421 & 0.084 & 0.210 & $0.0295^{*}$ & 0.03 & 1.89 & 2.106 & 0.675 & 0.259 & $0.024^{* *}$ & 1.04 & & \\
\hline $\begin{array}{l}\text { Anxiety symptoms } \\
\text { (HADS-A) }\end{array}$ & -0.141 & 0.018 & 0.272 & $0.0044 * *$ & 0.07 & 1.72 & $\mathrm{I} .873$ & 0.626 & 0.271 & $0.035^{*}$ & 1.24 & & \\
\hline
\end{tabular}

Notes: Adjusted model was applied using a stepwise linear regression. $* p<0.05, * * p<0.01$, $* * * p<0.001$.

Abbreviations: $\beta$, standardized regression coefficients; B, regression coefficient; HADS-A, Hospital Anxiety and Depression Scale-Anxiety symptoms; PTGI, Post-traumatic Growth Inventory; SE, standard error; VIF, variance inflation factor.

Table 3 Summary Of The Linear Regression Of Significant Predictors For PTSD In Patients With Breast Cancer

\begin{tabular}{|c|c|c|c|c|c|c|c|c|c|c|c|c|c|}
\hline \multirow[t]{2}{*}{ Variable } & \multicolumn{6}{|c|}{ Unadjusted Model } & \multicolumn{7}{|c|}{ Adjusted Model } \\
\hline & B & SE & $\beta$ & p-value & $\mathbf{R}^{2}$ & DW & B & SE & $\boldsymbol{\beta}$ & p-value & VIF & $\mathbf{R}^{2}$ & DW \\
\hline Chronic pain (score) & 0.076 & 0.011 & 0.498 & $<0.001 * * *$ & 0.243 & 1.93 & 0.049 & 0.012 & 0.316 & $<0.001 * * *$ & 1.11 & 0.40 & 1.96 \\
\hline Fatigue severity (BFI) & 0.016 & 0.005 & 0.304 & $0.00 I^{* *}$ & 0.084 & 2.13 & - & - & - & - & - & & \\
\hline Alexithymia (TAS) & 0.013 & 0.005 & 0.241 & $0.012^{*}$ & 0.049 & 2.20 & - & - & - & - & - & & \\
\hline Depression (PHQ-9) & 0.032 & 0.006 & 0.412 & $<0.001 * * *$ & 0.164 & 1.89 & - & - & - & - & - & & \\
\hline $\begin{array}{l}\text { Anxiety symptoms } \\
\text { (HADS-A) }\end{array}$ & 0.034 & 0.008 & 0.391 & $<0.001 * * *$ & 0.145 & 2.23 & 0.018 & 0.007 & 0.212 & $0.011 *$ & 1.19 & & \\
\hline $\begin{array}{l}\text { Cognition (Fact-Cog } \\
\text { total score) }\end{array}$ & -0.014 & 0.002 & -0.585 & $<0.001 * * *$ & 0.338 & 2.11 & -0.009 & 0.002 & -0.350 & $<0.00 I^{* * * *}$ & 1.30 & & \\
\hline Psychiatric history & 0.228 & 0.071 & 0.260 & $0.002 * *$ & 0.061 & 1.97 & - & - & & - & - & & \\
\hline
\end{tabular}

Notes: Adjusted model was applied using a stepwise linear regression. $* P<0.05, * * P<0.01, * * * P<0.001$.

Abbreviations: B, regression coefficient; BMI, body mass index; BFI, Brief Fatigue Inventory; HADS-A, Hospital Anxiety and Depression Scale-Anxiety symptoms; PHQ-9, Patient Health Questionnaire-9; SE, standard error; VIF, variance inflation factor. 
symptoms. No obvious multicollinearity between variables was observed based on the VIF range of 1.11-1.30. Furthermore, the Durbin-Watson test $(p s>0.05)$ suggested no significant autocorrelation.

\section{Discussion}

In this study, we found that patients with breast cancer varied regarding the development of PTSS and PTG. Some had PTG, whereas others had PTSS, and a weak association was found between PTG and PTSS. Furthermore, PTG and PTSS had distinct correlates. We observed that having a higher education level, better family support, more anxiety symptoms, and having had chemotherapy were associated with PTG. By contrast, severe depression symptoms and poor cognition were associated with PTSS.

Our results indicated a positive weak association between PTG and PTSS, which suggested a crucial underlying mechanism involving PTG and PTSS after trauma. However, the current literature has reported mixed findings regarding the association between PTG and PTSS. Nonetheless, our results are consistent with the findings of previous studies, ${ }^{8,33}$ even though some researchers did not find such associations in patients with breast cancer., $9,13,14$ However, several possible explanations do exist for the weak or absent association between PTG and PTSS. First, the presence of shared common causes of PTG and PTSS or some mediators linking PTG and PTSS might diminish the magnitude of their relationship. It has been proposed that deliberate rumination after a traumatic event ${ }^{34}$ and coping with social support ${ }^{15}$ are the common causes of PTG and PTSS. Furthermore, in the study by Morris and Shakespeare-Finch, the association between PTG and PTSS vanished when other predictors, such as rumination and social support, were included. Therefore, understanding that several factors may be involved in the mechanism between PTG and PTSS following trauma could help discern the complexity of PTG and PTSS.

Another possibility for the weak association between PTG and PTSS in our study is the underlying cultural stoicism in Taiwan and Asia. ${ }^{35}$ Self-disclosure has been demonstrated to have beneficial effects on PTSS and PTG. ${ }^{36}$ However, Taiwanese typically suppress their feelings and tend not to convey their experience. ${ }^{35}$ Moreover, a study found that individuals who tended not to disclose their traumatic events showed less growth and higher PTSS. ${ }^{37}$ Consequently, the nondisclosure owing to cultural differences may attenuate the association between PTG and PTSS.

Finally, the magnitude of the association between PTG and PTSS could have been moderated by the traumatic event of breast cancer stage. ${ }^{18,38}$ For example, a stronger association between PTG and PTSS was observed in children and the possible explanation could be that they are more profoundly affected by the traumatic experience, thereby having higher PTSS with a greater likelihood of PTG. ${ }^{39}$ It was also reported that the diversity of disease courses of cancer might have an impact on the development of post-traumatic growth. ${ }^{16}$ Nonetheless, additional studies are necessary to understand the possible moderators of PTG and PTSS, such as prolonged financial and psychological burden, poor prognoses, and poor outcomes, in the various clinical stages of breast cancer.

In our study, we found that higher years of education, better family support, anxiety symptoms, and having had chemotherapy were associated with PTG. No clear association was reported between the years of education and PTG in cancer survivors. ${ }^{40}$ Our results provide evidence regarding the beneficial effects of education on PTG, which is concordant with the results of a previous study. ${ }^{25}$ Although favorable family support was suggested to be involved in $\mathrm{PTG}^{41}$ few empirical studies have examined this correlation. Notably, our finding was concordant with that of a previous study, which demonstrated the beneficial effect of family support on PTG in patients with cancer. ${ }^{42}$ On the other hand, some studies have reported inconsistent findings, wherein they observed no association between family support and PTG. ${ }^{43,44}$ This ambiguity in the results warrant additional studies. Finally, although some researchers have reported regarding the positive or negative life changes after diagnosis and therapy, ${ }^{45}$ not much attention has been paid to the effect of chemotherapy on PTG. Nevertheless, our study results revealed that patients with breast cancer who received chemotherapy had higher PTG than those who did not. Patients with cancer who receive chemotherapy and cope successfully with the associated distress manifest better PTG. ${ }^{46}$

Chronic pain, anxiety symptoms, and poorer cognition were associated with the development of PTSS. Notably, two contrasting explanations exist regarding anxiety symptoms and chronic pain in PTSS. First, some researchers have proposed that anxiety symptoms and pain were a part of PTSS, ${ }^{47,48}$ and could be alleviated with PTSS intervention. ${ }^{49}$ Second, it is hypothesized that people with chronic pain and anxiety symptoms are more vulnerable to PTSS. Some 
longitudinal studies have supported this theory; ${ }^{49,50}$ although, our cross-sectional study cannot directly support this theory. Nonetheless, these results suggest a possible reciprocal relationship among chronic pain, anxiety symptoms, and PTSS. Notably, the significant association between poor cognition and PTSS observed in our study is consistent with the observation in the current literature. ${ }^{51,52}$

The perceived stress after trauma might substantially occupy the limited cognitive capacity of individuals ${ }^{53}$ and cause impairments in their information processing, such as recognition, attentional narrowing, and memory deficits. ${ }^{54}$ In addition, negative self-appraisals, traumatic events, and one's coping ability all considerably affect the perception of cognitive problems after trauma. ${ }^{55}$

\section{Implications}

Our study had some clinical implications. First, our results revealed that anxiety symptoms after trauma might enable patients with breast cancer to see new opportunities during PTG, besides resulting in more PTSS. Some factors could probably moderate and mediate the mechanisms of anxiety symptoms, PTG, and PTSS in patients with breast cancer. Finally, chronic pain and cognitive decline are common combinations in patients with breast cancer, especially for patients who receive chemotherapy. The management of pain, cognitive function examination, and rehabilitation should be considered in the clinical practice of oncology.

\section{Limitations}

This study had some limitations. First, the sample size was small, leading to a limited power of detection of some possible predictors. Second, because this was a cross-sectional study, the causal inference was limited. Finally, not all possible psychological and clinical correlates in the literature ${ }^{16}$ were included in this study.

\section{Data Sharing Statement}

The data that support the findings of this study are available from the corresponding author upon reasonable request.

\section{Acknowledgments}

This study was supported by grants from the Chang Gung Memorial Hospital, Chiayi, Taiwan (grant numbers: CORPG6G0101, CORPG6G0131). The funders had no role in the study design, data collection, and analysis, decision to publish, or preparation of the manuscript. Hong-Ming Chen and Vincent Chin-Hung Chen are cofirst authors for this study.

\section{Disclosure}

The authors report no conflicts of interest in this work.

\section{References}

1. Santiago PN, Ursano RJ, Gray CL, et al. A systematic review of PTSD prevalence and trajectories in DSM-5 defined trauma exposed populations: intentional and non-intentional traumatic events. PLoS One. 2013;8(4):e59236. doi:10.1371/journal.pone.0059236

2. Koopman C, Butler LD, Classen C, et al. Traumatic stress symptoms among women with recently diagnosed primary breast cancer. $J$ Trauma Stress. 2002;15(4):277-287. doi:10.1023/A:1016295610660

3. Cordova MJ, Riba MB, Spiegel D. Post-traumatic stress disorder and cancer. Lancet Psychiatry. 2017;4(4):330-338. doi:10.1016/S22150366(17)30014-7

4. Swartzman S, Booth JN, Munro A, et al. Posttraumatic stress disorder after cancer diagnosis in adults: a meta-analysis. Depress Anxiety. 2017;34(4):327-339. doi:10.1002/da.22542

5 . Tedeschi R, Calhoun L. The posttraumatic growth inventory: measuring the positive legacy of trauma. J Trauma Stress. 1996;9(3):455471. doi: $10.1002 /$ jts. 2490090305

6. Tedeschi R, Calhoun LG. Posttraumatic growth: conceptual foundations and empirical evidence. Psychol Inq. 2004;15(1):1-18. doi:10.1207/s15327965pli1501_01

7. Coroiu A, Körner A, Burke S, et al. Stress and posttraumatic growth among survivors of breast cancer: a test of curvilinear effects. Int $J$ Stress Manag. 2016;23(1):84. doi:10.1037/a0039247

8. Groarke A, Curtis R, Groarke JM, et al. Post-traumatic growth in breast cancer: how and when do distress and stress contribute? Psychooncology. 2017;26(7):967-974. doi:10.1002/pon.v26.7

9. Chan MW, Ho SM, Tedeschi RG, et al. The valence of attentional bias and cancer-related rumination in posttraumatic stress and posttraumatic growth among women with breast cancer. PsychoOncology. 2011;20(5):544-552. doi:10.1002/pon.1761

10. McDonough MH, Sabiston CM, Wrosch C. Predicting changes in posttraumatic growth and subjective well-being among breast cancer survivors: the role of social support and stress. Psycho-Oncology. 2014;23(1):114-120. doi:10.1002/pon.v23.1

11. Shand LK, Cowlishaw S, Brooker JE, et al. Correlates of post-traumatic stress symptoms and growth in cancer patients: a systematic review and meta-analysis. Psycho-Oncology. 2015;24(6):624-634. doi:10.1002/pon.3719

12. Koutrouli N, Anagnostopoulos F, Potamianos G. Posttraumatic stress disorder and posttraumatic growth in breast cancer patients: a systematic review. Women Health. 2012;52(5):503-516. doi:10.1080/ 03630242.2012.679337

13. Cordova MJ, Cunningham LL, Carlson CR, et al. Posttraumatic growth following breast cancer: a controlled comparison study. Health Psychol. 2001;20(3):176. doi:10.1037/0278-6133.20.3.176

14. Ho SM, Chan MW, Yau T, et al. Relationships between explanatory style, posttraumatic growth and posttraumatic stress disorder symptoms among Chinese breast cancer patients. Psychol Health. 2011;26 (3):269-285. doi:10.1080/08870440903287926

15. Morris BA, Shakespeare-Finch J. Rumination, post-traumatic growth, and distress: structural equation modelling with cancer survivors. Psycho-oncology. 2011;20(11):1176-1183. doi:10.1002/pon.1827

16. Casellas-Grau A, Ochoa C, Ruini C. Psychological and clinical correlates of posttraumatic growth in cancer: a systematic and critical review. Psychooncology. 2017;26(12):2007-2018. doi:10.1002/pon. v26.12

17. Parikh D, De Ieso P, Garvey G, et al. Post-traumatic stress disorder and post-traumatic growth in breast cancer patients-A systematic review. Asian Pac J Cancer Prev. 2015;16(2):641-646. doi:10.7314/ APJCP.2015.16.2.641 
18. Tedeschi RG, Calhoun LG, Cann A. Evaluating resource gain: understanding and misunderstanding posttraumatic growth. Appl Psychol. 2007;56(3):396-406. doi:10.1111/apps.2007.56.issue-3

19. Bellizzi KM, Smith AW, Reeve BB, et al. Posttraumatic growth and health-related quality of life in a racially diverse cohort of breast cancer survivors. J Health Psychol. 2010;15(4):615-626. doi:10.1177/ 1359105309356364

20. Tjemsland L, Soreide JA, Malt UF. Posttraumatic distress symptoms in operable breast cancer III: status one year after surgery. Breast Cancer Res Treat. 1998;47(2):141-151. doi:10.1023/A:1005957302990

21. Bleiker EM, Pouwer F, van der Ploeg HM, et al. Psychological distress two years after diagnosis of breast cancer: frequency and prediction. Patient Educ Couns. 2000;40(3):209-217. doi:10.1016/ S0738-3991(99)00085-3

22. Chan CMH, Wan Ahmad WA, MD Yusof M, et al. Effects of depression and anxiety on mortality in a mixed cancer group: a longitudinal approach using standardised diagnostic interviews. Psycho-Oncology. 2015;24(6):718-725. doi:10.1002/pon.3714

23. Pilevarzadeh M, Amirshahi M, Afsargharehbagh R, et al. Global prevalence of depression among breast cancer patients: a systematic review and meta-analysis. Breast Cancer Res Treat. 2019;176 (3):519-533. doi:10.1007/s10549-019-05271-3

24. Jim HS, Andrykowski MA, Munster PN, et al. Physical symptoms/side effects during breast cancer treatment predict posttreatment distress. Ann Behav Med. 2007;34(2):200-208. doi:10.1007/BF02872674

25. Danhauer SC, Case LD, Tedeschi R, et al. Predictors of posttraumatic growth in women with breast cancer. Psychooncology. 2013;22 (12):2676-2683. doi:10.1002/pon.v22.12

26. Chen $\mathrm{CH}$, Shen WW, Tan $\mathrm{HK}$, et al. The validation study and application of stratum-specific likelihood ratios in the Chinese version of SPAN. Compr Psychiatry. 2003;44(1):78-81. doi:10.1053/ comp. 2003.50013

27. Taylor GJ, Bagby RM, Parker JDA. The 20-item toronto alexithymia scale: IV. Reliability and factorial validity in different languages and cultures. J Psychosom Res. 2003;55(3):277-283. doi:10.1016/S00223999(02)00601-3

28. Mendoza TR, Wang XS, Cleeland CS, et al. The rapid assessment of fatigue severity in cancer patients: use of the brief fatigue inventory. Cancer. 1999;85(5):1186-1196. doi:10.1002/(ISSN)1097-0142

29. van Hout B, Janssen MF, Feng YS, et al. Interim scoring for the EQ5D-5L: mapping the EQ-5D-5L to EQ-5D-3L value sets. Value Health. 2012;15(5):708-715. doi:10.1016/j.jval.2012.02.008

30. Cheung Y, Lim SR, Shwe M, et al. Psychometric properties and measurement equivalence of the English and Chinese versions of the functional assessment of cancer therapy-cognitive in Asian patients with breast cancer. Value Health. 2013;16(6):1001-1013. doi:10.1016/j.jval.2013.06.017

31. Carlsson AM. Assessment of chronic pain. I. Aspects of the reliability and validity of the visual analogue scale. Pain. 1983;16(1):87101. doi:10.1016/0304-3959(83)90088-X

32. O'brien RM. A caution regarding rules of thumb for variance inflation factors. Qual Quant. 2007;41(5):673-690. doi:10.1007/s11135006-9018-6

33. Morrill EF, Brewer NT, O'Neill SC, et al. The interaction of posttraumatic growth and post-traumatic stress symptoms in predicting depressive symptoms and quality of life. Psychooncology. 2008;17 (9):948-953. doi:10.1002/pon.v17:9

34. Triplett KN, Tedeschi RG, Cann A, et al. Posttraumatic growth, meaning in life, and life satisfaction in response to trauma. Psychol Trauma. 2012;4(4):400. doi:10.1037/a0024204

35. Yang K-S. Social orientation and individual modernity among Chinese students in Taiwan. J Soc Psychol. 1981;113(2):159-170. doi:10.1080/00224545.1981.9924368

36. Tedeschi RG, McNally RJ. Can we facilitate posttraumatic growth in combat veterans? Am Psychol. 2011;66(1):19. doi:10.1037/a0021896
37. Taku K, Tedeschi RG, Cann A, et al. The culture of disclosure effects of perceived reactions to disclosure on posttraumatic growth and distress in Japan. J Soc Clin Psychol. 2009;28(10):1226-1243. doi:10.1521/jscp.2009.28.10.1226

38. Liu AN, Wang LL, Li HP, et al. Correlation between posttraumatic growth and posttraumatic stress disorder symptoms based on pearson correlation coefficient: a meta-analysis. J Nerv Ment Dis. 2017;205 (5):380-389. doi:10.1097/NMD.0000000000000605

39. Alisic E, Van der Schoot T, van Ginkel JR, et al. Looking beyond posttraumatic stress disorder in children: posttraumatic stress reactions, posttraumatic growth, and quality of life in a general population sample. J Clin Psychiatry. 2008;69(9):1455-1461. doi:10.4088/ JCP.v69n0913

40. Jim HS, Jacobsen PB. Posttraumatic stress and posttraumatic growth in cancer survivorship: a review. Cancer J. 2008;14(6):414-419. doi:10.1097/PPO.0b013e31818d8963

41. Berger R, Weiss T. The posttraumatic growth model: an expansion to the family system. Traumatology. 2009;15(1):63-74. doi:10.1177/ 1534765608323499

42. Tanriverd D, Savas E, Can G. Posttraumatic growth and social support in Turkish patients with cancer. Asian Pac J Cancer Prev. 2012;13(9):4311-4314. doi:10.7314/APJCP.2012.13.9.4311

43. Taku K. Relationships among perceived psychological growth, resilience and burnout in physicians. Pers Individ Dif. 2014;59:120-123. doi:10.1016/j.paid.2013.11.003

44. Senol-Durak E, Ayvasik HB. Factors associated with posttraumatic growth among myocardial infarction patients: perceived social support, perception of the event and coping. J Clin Psychol Med Settings. 2010;17(2):150-158. doi:10.1007/s10880-010-9192-5

45. Arpawong TE, Richeimer SH, Weinstein F, et al. Posttraumatic growth, quality of life, and treatment symptoms among cancer chemotherapy outpatients. Health Psychol. 2013;32(4):397-408. doi: $10.1037 / \mathrm{a} 0028223$

46. Bussell VA, Naus MJ. A longitudinal investigation of coping and posttraumatic growth in breast cancer survivors. J Psychosoc Oncol. 2010;28(1):61-78. doi:10.1080/07347330903438958

47. Benedict TM, Singleton MD, Nitz AJ, et al. Effect of chronic low back pain and post-traumatic stress disorder on the risk for separation from the US army. Mil Med. 2019. doi:10.1093/milmed/usz020

48. Dai W, Kaminga AC, Tan H, et al. Comorbidity of post-traumatic stress disorder and anxiety in flood survivors: prevalence and shared risk factors. Medicine (Baltimore). 2017;96(36):e7994. doi:10.1097/ MD.0000000000007994

49. Church D. Reductions in pain, depression, and anxiety symptoms after PTSD remediation in veterans. Explore. 2014;10(3):162-169. doi:10.1016/j.explore.2014.02.005

50. Ciccone DS, Kline A. A longitudinal study of pain and pain catastrophizing in a cohort of National guard troops at risk for PTSD. PAIN $^{\circledR}$. 2012;153(10):2055-2060. doi:10.1016/j.pain.2012.06.015

51. Qureshi SU, Long ME, Bradshaw MR, et al. Does PTSD impair cognition beyond the effect of trauma? J Neuropsychiatry Clin Neurosci. 2011;23(1):16-28. doi:10.1176/appi.neuropsych.23.1.16

52. Schuitevoerder S, Rosen JW, Twamley EW, et al. A meta-analysis of cognitive functioning in older adults with PTSD. J Anxiety Disord. 2013;27(6):550-558. doi:10.1016/j.janxdis.2013.01.001

53. Aggarwal NT, Wilson RS, Beck TL, et al. Perceived stress and change in cognitive function among adults aged 65 and older. Psychosom Med. 2014;76(1):80. doi:10.1097/PSY.0000000000000016

54. Buckley TC, Blanchard EB, Neill WT. Information processing and ptsd: a review of the empirical literature. Clin Psychol Rev. 2000;20 (8):1041-1065. doi:10.1016/S0272-7358(99)00030-6

55. Samuelson KW, Bartel A, Valadez R, et al. PTSD symptoms and perception of cognitive problems: the roles of posttraumatic cognitions and trauma coping self-efficacy. Psychol Trauma. 2017;9 (5):537-544. doi:10.1037/tra0000210 


\section{Publish your work in this journal}

Neuropsychiatric Disease and Treatment is an international, peerreviewed journal of clinical therapeutics and pharmacology focusing on concise rapid reporting of clinical or pre-clinical studies on a range of neuropsychiatric and neurological disorders. This journal is indexed on PubMed Central, the 'PsycINFO' database and CAS, and is the official journal of The International Neuropsychiatric Association (INA). The manuscript management system is completely online and includes a very quick and fair peer-review system, which is all easy to use. Visit http://www.dovepress.com/testimonials.php to read real quotes from published authors. 